\title{
Exposomics and Growth Rings in the Teeth
}

\author{
Michael AB Naafs* \\ International Health Consultancy, Dutch Internist Endocrinologist, Netherlands
}

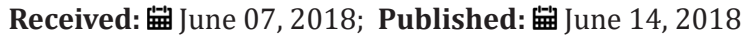

*Corresponding author: Michael AB Naafs, Dutch Internist Endocrinologist, Health Consultant at Naafs,International Health Consultancy, Rhodoslaan 20, 7577KN, Oldenzaal, Netherlands

\begin{abstract}
In this mini-review recent developments in dental exposomic fingerprinting and their association with neurodevelopmental disorders as the autism spectrum dosorders (ASD) are discussed.Sophisticated microspatial techniques enable researchers to read the growth rings of deciduous teeth like the growth rings of a tree.Dysregulations in fetal zinc-copper metabolism found in this way appear to predict ASD in later life with 90 percent accuracy.
\end{abstract}

\section{Introduction}

The 'exposome' concept was introduced in 2005 to address the disparity between the genomic sciences,where rapid technological advances provided an expanse of high-precision analyses, and the environmental exposure sciences that measured a small fraction of the thousands of environmental exposures individuals experience [1]. The exposome concept encompasses lifecourse environmental exposures (including lifestyle factors),from the prenatal period onwards.It is important to consider that the exposure includes not only external exposures but also internal factors (e.g. inflammation,infection and the microbiome) [2].

While the definition of the exposome evolves, the fundamental concept continues to gain international momentum [3]. The exposome,unlike the genomic sequence is highly variable and continues throughout the individual's lifetime [4]. Longitudinal birth cohort studies collecting biomarkers of environmental chemical exposure during pregnancy and then follow-up offspring into later life are time consuming and expensive.Another problem in studying the fetal exposome is that maternal biomarkers do not reflect fetal exposure for all chemicals.Reliance on maternal biomarkers of fetal exposure do not necessarily provide accurate measures of fetal exposure for all chemicals,as variability in placental transpot and metabolism can be overlooked [5-7].

Umbillical cord blood has been successfully collected at birth in epidemiologic studies and has provided valuable exposure information.However, for compounds with a short half-life in blood,cord blood can only provide on the latter part of the trimester [8-11]. For decades teeth have been used to evaluate long-term cumulative exposure to metals. Recently developed

high-dimensional analytical methods that combine sophisticated histological and chemical analysis to precisely sample tooth layers that correspond to specific life stages, showed the potential to reconstruct the exposome in the second and third trimester of prenatal development and during early childhood [12]. In this minireview recent developments in dental exposomic fingerprinting and their association with neurodevelopmental disorders as the autism spectrum disorders (ASD) are discussed.

\section{Dental Exposomic Fingerprinting}

Recently,deciduous teeth have been proposed as a promising biomatrix for exposomics analysis for several reasons [12]. Single deciduous tooth contains both prenatal and postnatal exposure information.Futhermore,due to specific growth patterns of dentine in deciduous teeth,chemicals accumulated in prenatal and postnatal periods are spatially separated by the neonatal line which is formed at birth.In addition,exposure chemicals can remain stable in the mineralized dental tissues.Metallomics has been used mostly so far in dental exposomic fingerprinting, but tracing organic chemicals in deciduous teeth is upcomng now $[13,14]$.

\section{Metallomics}

Metals have been measured in teeth for many decades,with lead being the most studied toxicant in the teeth [15-17]. The skeletal comparment comprises the major depository of total lead burden and is also a potent source of internal exposure,due to release of lead during bone remodelling,such as occurs in pregnancy and osteoporosis [18]. Over the last two decades microspatial samplng with sophisticated histologic analysis has provided a means to 
uncover the timing of metal uptake, including prenatal exposure from teeth biomarkers.

Various techniques are in use as e.g. inductively coupled plasma mass spectrometry (ICP-MS), neutron activation analysis (NAA), fluorescent synchotron radiation X-ray fluorescence (SR-XFR),XFR tomography energy dispersive X-ray (EDX), proton induced X-ray emission (PIXE), laser ablation (LA-ICP-MS) and secondary ion mass spectrometry (SIMS). Fourier transform-infrared (FT-IR) and Raman spectroscopy are excellent tools for molecular mapping [19]. With automation of arraying small samples,rapid data collection of multiple low-volume and-concentration samples, together with data reduction and analysis, achieving high-throughput techniques is possible now [19].

Detailed validation against environmental samples and other biomatrices has only been performed in the last 5 years [20-25]. For validation of Mn (manganese) there was a significant positive association of levels in parts of dentine formed in the second trimester with Mn loading in floor dust sampled during the second trimester of pregnancy [26]. That study also showed that Mn levels in dentine adjacent the neonatal line was strongly associated with cord blood Mn concentrations,both biomarkers reflecting $\mathrm{Mn}$ uptake close tot he time of birth [26]. The isotopic partitioning of other elements ( $\mathrm{Mg}, \mathrm{Fe}, \mathrm{Cu}$ and $\mathrm{Zn}$ ) from diet to tissue is also under investigation now.In particular, investigations in copper and zinc metabolism revealed marked results,drawing attention in mainstream press,because of a link with autism spectrum disorder (ASD) and possibly ADHD (attention deficit hyperactivity disorder) $[27,28]$.

\section{Metallomics and ASD}

Using evidence found in baby teeth,researchers from the Institute for Exposomic Research at the Icahn School of Medicine at Mount Sinai,New York,report that cycles involved in zinc and copper metabolism are dysregulated in autism spectrum disorders (ASD),and can be used to predict who will later develop the disease. The researchers used the teeth to reconstruct prenatal and earlylife exposures to nutrient and toxic elements [29].

This is the first study in the world to generate a 90 percent accurate fetal and early childhood biomarker of ASD using a longitudinal analysis of distinct metabolic pathways,and to replicate it in four independent study populations. The results of this research could produce a new diagnostic approach for ASD early in life before the disorder appears and could catalyze new treatments and prevention strategies [30].

About 1 in 68 children has been identified with ASD, according to the CDC (Centers for Disease Control and Prevention).To determine the effect of zinc and copper metabolism Mount Sinai researchers used biomarkers in baby teeth collected from a twin study running in Sweden and replicated these findings in three other populations: a group of non-twin siblings in New York,and two populations of non-related participants from Texas and the United Kingdom.

During fetal and chilldhood development, a new tooth layer is formed every day.As each of these "growth rings"forms an inprint of many of the chemicals circulating in the body as captured in each layer,which provides a chronological record of exposure,as mentioned above. The research team used lasers (LA-ICP-MS) to sample these layers and reconstruct the past exposures along incremental markngs,similar to using growth rings on a tree,to determine the tree's growth history.

The authors found significant divergences between ASD-affected children and their healthy siblings and used these biomarkers to predict the emergence of disease [29]. These findings suggest that the cyclic metabolism of nutrients and toxicants is critical in healthy neurodevelopment,and the emergence of autism. Future studies of metal metabolic cycles to study the association with ADHD and other neurodevelopmental disorders are planned [29].

\section{Conclusion}

Exposomics is a science in its infancy,introduced as a concept in 2005.However,just a decade later this concept has gained international momentum with several known research groups in e.g. the U.S.,U.K and Sweden.Sophisticated microspatial techniques make tracing of metals,elements and organics possible in biomatrices as deciduous teeth.It appears that deciduous teeth form growth rings during fetal life and postnatal, reflecting an inprint of e.g. accumulated metals from maternal diet or other sources.In this way the dental exposome can be read as the growth rings of a tree.Very recently, it has been shown by Mount Sinai researchers in New York that dysreguations in zinc-copper metabolism predict later development of autism spectrum disorders (ASD),This is the world's first study that provides a biomarker predicting ASD with a 90 percent accuracy.Future studies investigating similar possible associations with ADHD are planned.Dental exposomic fingerprinting has a bright future,as this landmark study shows [29].

\section{References}

1. Wild CP (2005) Complementing the genome with an "exposome": the outstandng challenge of environmental exposure measurement in molecular epidemiology.Cancer Epidemiol. Biomarkers Prev 14(8): 1847-1850.

2. Rappaport SM, Smith MT (2010) Epidemiology, Environment And Disease Risks. Science 330 (6003): 460-461.

3. Miller GW, Jones D (2014) The nature of nurture:refining the definition of the exposome. Toxicol.Sci 137(1): 1-2.

4. Wild C.P (2012) The exposome:from concept to utility. Int J Epidemiol 41(1): 24-32.

5. Needhan LL,Grandjean P, Heinzow B, Poul J Jørgensen, Flemming Nielsen, et al. (2011) Partitiion of environmental chemicals between maternal and fetal blood and tissues. Environ Sci Technol 45(3): 1121-1126.

6. Rudge C, Rollin HB, Naguirea CM, Thomassen Y, Rudge MC, et al. (2009) The placenta as a barrier for toxic and essential elements in paired maternal and cord blood samples of South African delivering women.J 
Environ Monit 11(7): 1322-1330.

7. Yoon M, Nong A, Clewell HJ, Taylor MD, Dorman DC, et al. (2009) Evaluating placental transfer and tissue concentrations of manganese in the pregnan rat and fetuses after inhalation with a PBPK model. Toxicol Sci 112(1): 44-58.

8. Aylward LL, Hays SM, Kiman CP, Marchitti SA, Kenneke JF, et al. (2014) Relationships of chemical concentrations in maternal and cord blood: a review of available data. J Toxicol Environ Health B Crit Rev 17(3): 175203.

9. Cooke GM (2014) Biomonitoring of human fetal exposure to environmental chemicals in early pregnancy. J Toxicol Environ Health B Crit Rev 17(4): 205-224.

10. Delvaux I,van Cauwenberghe J, Den Hond E, Schoeters G, Govarts E, et al. (2014)Prenatal exposure to environmental contaminants and body composition at age 7-9 years. Environ Res 132: 24-32.

11. Lin CC, Chen YC, Su FC, Lin CM, Liao HF, et al. (2013) In utero exposure to environmental lead and manganese and neurodevelopment at 2 years of age. Environ Res 123: 52-57.

12. Andra SS, Austin C, Arora M (2016) The tooth exposome in children:s health research. Curr Opin Pediatr 28(2): 221-227.

13. Vermeulen R (2016) Use of Metabolomics to Advance Research on Environmental Exposures and the Human Exposome Workshop in Brief. Washington DC National Academic Press, USA Mar 22.

14. Lee Y, Seo E, Park TM, Yin Ning Chiang†, Klaus Dreisewerd, et al. (2017) Analysis of Lipids in Deciduous Teeth by Matrix-Assisted Laser Desorption-Ionization Mass Spectrometry (MALDI MS) Mass Spectrometry Letters 8(4): 11086-11092.

15. Altshuller L, Halak DB, Landing BI (1962) Deciduous teeth as an index of body burden of lead.J.Pediatrics 60(2): 224-229.

16. Needleman HL, Shapiro IM (1974) Dentine lead levels in asymtomatic Philidelphia school children:subclinical exposure in high and low risk groups. Environ Health Persp 7: 27-31.

17. Needleman HL, Davidson I, Sewell M (1974) Subclinical lead exposure in Philadelphia schoolchildren:identification by dentine lead analysis.N Engl J Med 290 (5): 245-248.
18. Hu H (1998) Bone lead as a new biologic marker of lead dose: recent findings and implications for public health. Environ Health Persp 106(4): 961-967.

19. Li YF, Qu Y, Chen C (2008) Metallonomics,elementonomics and analytical techniques.Pure Appl.Chem. 80(12): 2577-2594.

20. Ericson JE (2001) Enamel lead biomarker for prenatal exposure assessment. Environ Res 87(3): 136-140.

21. Ericson JE, Rinderknecht A, Gonzalez EJ, Francis M.CrinellabMichael T.Kleinman, et al. (2001) Meaurement of manganese with respect to calcium in histological enamel cross sections: toward a new manganese biomarker. Environ Res 86(1): 46-50.

22. Arora M, Chan SW, Kennedy BJ, AshwiniSharma, DanteCrisante, et al. (2004) Spatial distribution of lead in the roots of human primary teeth. J Trace Elem Med Biol 18(2):135-39.

23. Arora M, Chan SW, Ryan CG, Kennedy BJ, Walker DM, et al. (2005) Spatial distribution of lead in enamel and coronal dentine of wistar rats. Biol Trace Elem Res 105(3): 159-170.

24. Arora M, Hare D, Austin C, Smith DR, Doble P, et al. (2011) Spatial distribution of manganese in enamel and coronal dentine of human primary teeth. Sci Total Environ 409(7): 1315-1319.

25. Arora M, Kennedy BJ, Ehlou S, Pearson NJ, Walker DM, et al. (2006) Spatial distribution of lead in human primary teeth as a biomarker of pre-and neonatal lead exposure. Sci Total Environ 37(1-3): 55-62.

26. Arora M, Asa Bradman, Christine Austin, Michelle Vedar, Nina Holland, et al. (2012) Determining fetal manganese exposure from mantle dentine of deciduous teeth. Environ Sci Technol 46(9): 5118-5125.

27. Dental Discovries: Teeth Contain Growth Rings Like Trees.

28. Baby teeth may predict autism, says study, Dental Nursing.

29. Curtin P, Austin C, Curtin A (2018) Dynamic features in feta land postnatal zinc-copper metabolic cycles predict the emergence of autism spectrum disorders. Science Advances 4(5).

30. Clues to Autism Found in Baby Teeth.Bioscience Technology.

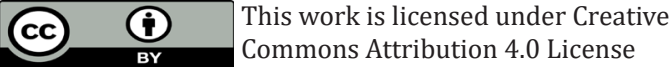

To Submit Your Article Click Here:

Submit Article
DOI: 10.32474/MADOHC.2018.02.000145

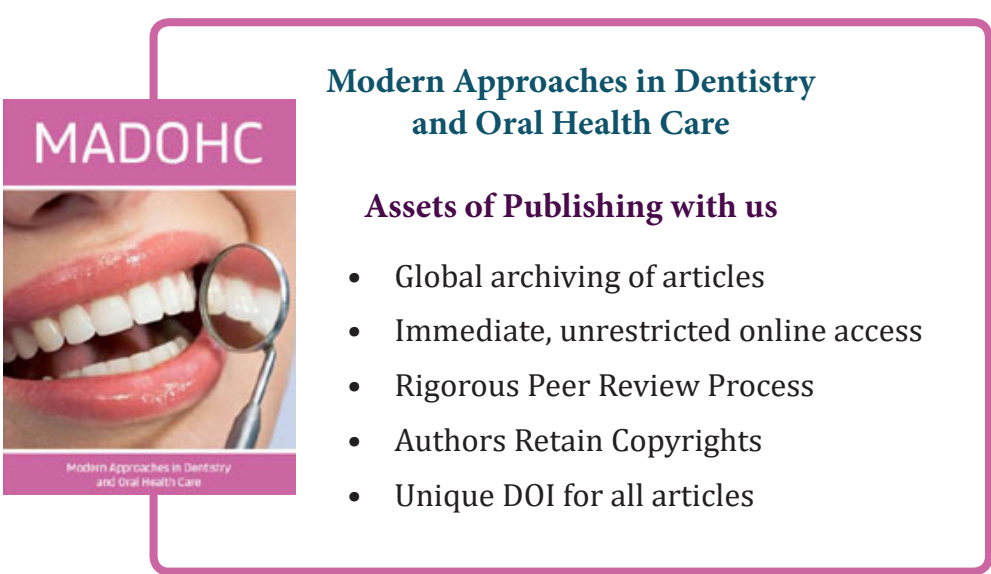

